\title{
Using the Edmonton Obesity Staging System in the real world: a feasibility study based on cross-sectional data
}

\author{
Rukia Swaleh MD, Taylor McGuckin MSc, Tyler W. Myroniuk PhD, Donna Manca MD MClSc, \\ Karen Lee MD MHSc, Arya M. Sharma MD DSc, Denise Campbell-Scherer MD PhD, \\ Roseanne O. Yeung MD MPH
}

\section{Abstract}

Background: The Edmonton Obesity Staging System (EOSS) combined with body mass index (BMI) enables improved functional and prognostic assessment for patients. To facilitate application of the EOSS in practice, we aimed to create tools for capturing comorbidity assessments in electronic medical records and for automating the calculation of a patient's EOSS stage.

Methods: In this feasibility study, we used cross-sectional data to create a clinical dashboard to calculate and display the relation between BMI and EOSS and the prevalence of related comorbidities. We obtained data from the Northern Alberta Primary Care Research Network and the Canadian Primary Care Sentinel Surveillance Network (CPCSSN). We included patients at least 18 years of age with BMI between 30 and 60 who visited a network clinic between July 2016 and July 2019. We calculated descriptive statistics and used stepwise ordinary least squares regression to assess the contributions of age, sex and BMI to EOSS variation.

Results: We created a clinical dashboard using the CPCSSN data presentation tool. Of the total 31496 patients included in the study, 23460 had a BMI of at least 30; BMI was unavailable for 8036 patients. Within each EOSS disease severity stage, there were similar proportions of patients from each BMI class (e.g., patients with EOSS stage 2 included $51.8 \%$ of those with BMI class I, $55.3 \%$ of those with BMI class II and $58.8 \%$ of those with BMI class III).

Interpretation: Using data from primary care electronic medical records, it was feasible to create a clinical dashboard for obesity that highlighted the severity and stage of obesity. Making this information easily accessible for individual clinical care and practice-level quality improvement may advance obesity care.

besity is a chronic disease that is defined as abnormal or excess adiposity causing physical or metabolic harm. ${ }^{1}$ The 2020 Canadian clinical practice guideline for obesity in adults ${ }^{2}$ highlighted the need for comprehensive clinical assessment of people living with obesity, including the use of both obesity classification (based on body mass index [BMI]) and stage of disease (based on the Edmonton Obesity Staging System [EOSS]) (Figure 1). ${ }^{3,4}$ The EOSS is a clinical tool that evaluates obesity-related comorbidities according to a 5-stage scale; staging on this scale has been demonstrated to correlate with clinical outcomes..$^{3,5-7}$

The EOSS allows assessment of the effect of obesityrelated comorbidities on individuals, beyond weight. ${ }^{5}$ These comorbidities drive increases in morbidity, mortality and health system costs for people living with obesity. ${ }^{8-12}$ Advancing care for those living with obesity requires efficient access to point-of-care tools for collating and synthesizing increasingly complex information at both the individual and the practice level. ${ }^{3}$
Applying the EOSS in routine clinical practice is hampered by the lack of standardized, user-friendly EOSS tools that take advantage of data from electronic medical records. ${ }^{3}$ In this pragmatic clinical feasibility study, we aimed to use data from

Competing interests: For activities outside the scope of the current study, Karen Lee has received consulting fees from Alberta Health Services, United Network Studio, Christenson Group of Companies and International WELL Building Institute, as well as honoraria for conference presentations and panels from the Canadian Society of Hospital Pharmacists, the Canadian Cardiovascular Pharmacists Network and the Canadian Institute of Public Health Inspectors; Arya Sharma has received personal fees from Novo Nordisk and Bausch Pharmaceuticals; Denise Campbell-Scherer has received an unrestricted educational grant from Novo Nordisk and has served on an obesity-related advisory panel for Pfizer; and Roseanne Yeung has received personal fees from Merck, Diabetes Canada, Novo Nordisk and Sanofi, as well as grants from Astra Zeneca and Allergen. No other competing interests were declared.

This article has been peer reviewed.

CMAJ Open 2021 December 7. DOI:10.9778/cmajo.20200231 
electronic medical records to develop case definitions for obesity-related comorbidities used in the EOSS disease severity stage; to determine the availability of suitable electronic data to calculate EOSS stages and identify data limitations; to describe the relation between BMI and EOSS, as well as the prevalence of obesity-related comorbidities in the study population; and to create a clinical dashboard to visualize obesity classification and staging, as well as relevant comorbidities, to support clinicians' efforts to improve care for people living with obesity.

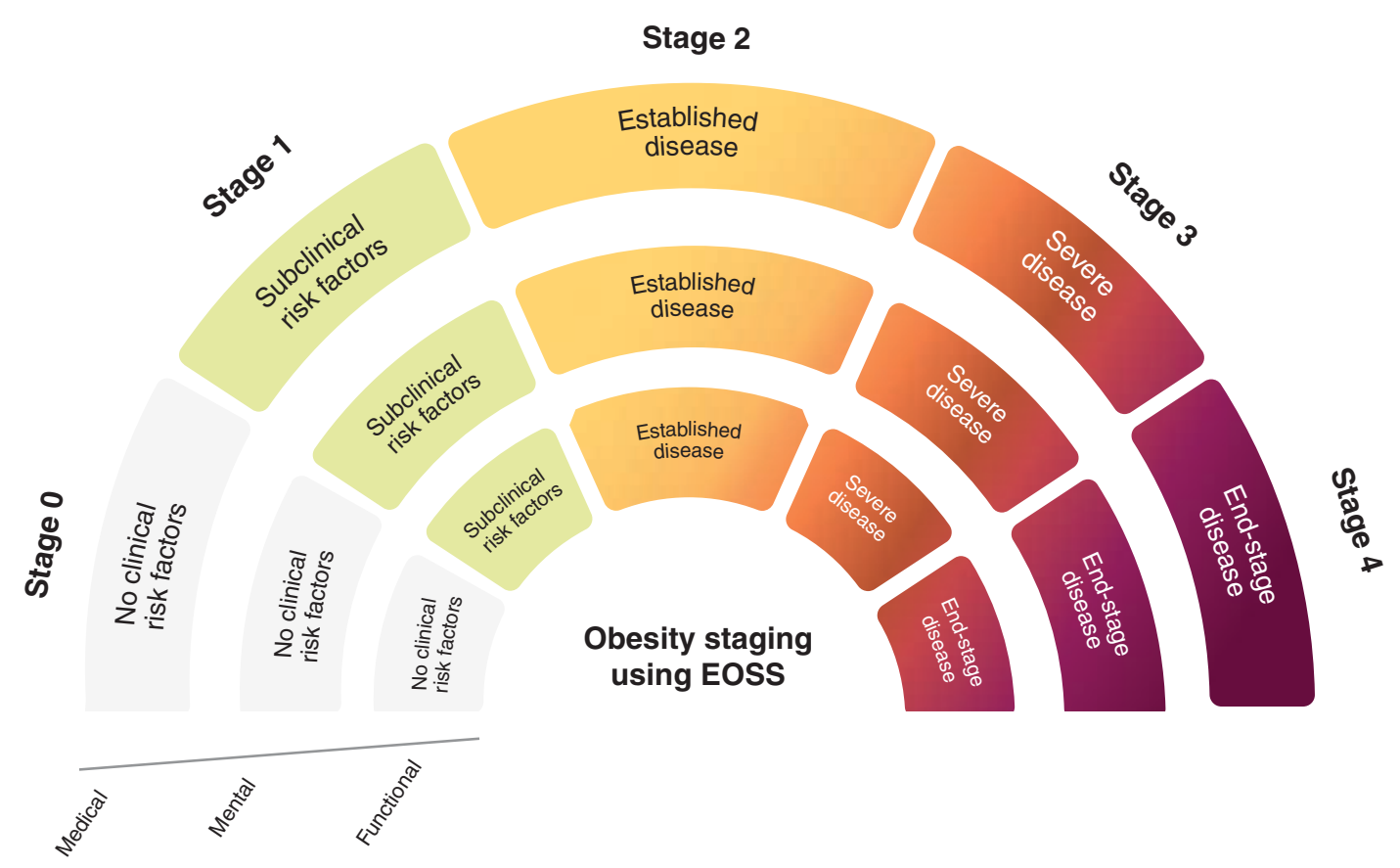

Obesity staging using recommended classification of BMI

\begin{tabular}{lc} 
Category & $\begin{array}{c}\text { Body mass } \\
\text { index }\end{array}$ \\
\hline Underweight & $<18.5$ \\
\hline Normal (healthy weight) & $18.5-24.9$ \\
\hline Overweight & $25-29.9$ \\
\hline Obesity class I & $30-34.9$ \\
\hline Obesity class II & $35-39.9$ \\
\hline Obesity class III & $\geq 40$
\end{tabular}

In people of South, Southeast or East Asian ethnicity metabolic risk is observed at lower BMI values. modified, with permission, from Atlantis and colleagues, ${ }^{3}$ and table component modified, with permission, from Rueda-Clausen and colleagues. ${ }^{4}$ 


\section{Methods}

\section{Setting and study design}

This population-based feasibility study involved a partnership between the Physician Learning Program ${ }^{13}$ and the Northern Alberta Primary Care Research Network (NAPCReN) ${ }^{14}$ which is 1 of 13 primary care research networks in Canada contributing data from electronic medical records to the Canadian Primary Care Sentinel Surveillance Network (CPCSSN). ${ }^{15}$ Our setting included 80 clinicians across 18 cities in Alberta, Canada, with 103153 patients contributing to the NAPCReN network.

The CPCSSN is Canada's first multidisease medical record surveillance system providing opportunities to inform and advance primary care.$^{15}$ With a national reach and an ability to consolidate information for several electronic medical record systems, the CPCSSN was suitable for creating the EOSS tool and enabled incorporation into practice of recommendations from the 2020 Canadian obesity guideline for adults. ${ }^{2}$ The Physician Learning Program, which is funded by the Alberta government, aligns with health system stakeholders to serve as an implementation hub to integrate best-available evidence and data on problems of clinical importance, and to co-create sustainable solutions to advance practice. ${ }^{13}$

As distinct from a classical epidemiologic study, this clinical feasibility study entailed recognition that variation in clinical practice is warranted, given that not all clinical measurements are indicated for all patients. As such, we expected some data to be unavailable.

\section{Study population}

We included individuals 18 years of age or older with BMI of at least 30 and at least 1 visit with a family physician contributing data to the NAPCReN between July 1, 2016, and July 1, 2019. We excluded patients with BMI above 60 because most patients with BMI in this range have mechanical and functional issues requiring tertiary care.

\section{Obesity-related comorbidities and EOSS staging}

We used validated CPCSSN case definitions for hypertension, diabetes and osteoarthritis. ${ }^{16}$ The definitions for the other comorbidities were informed by Padwal and colleagues. ${ }^{5}$ Where additional granularity was needed or no validated disease case definition was available, our team of clinicians and researchers identified clinical practice guidelines ${ }^{17,18}$ and created pragmatic case definitions based on available data. These sources included a combination of laboratory codes, Anatomical Therapeutic Chemical (ATC) codes for medications and the International Classification of Diseases, Ninth Revision, Clinical Modification. The data definitions for the EOSS comorbidities are included in Appendix 1, available at www.cmajopen.ca/content/9/4/E1141/ suppl/DC1.

The EOSS includes stages from 0 to 4 , with the stage being based on the most severely affected obesity-related comorbidity. Padwal and colleagues ${ }^{5}$ showed that a higher stage indicates a higher risk of death. We assigned an EOSS stage for each comorbidity, and then assigned each individual's overall EOSS stage as the highest stage of all their comorbidities. For example, if a person had stage 2 for liver disease, stage 1 for hypertension and stage 0 for kidney disease, the overall EOSS stage was deemed to be 2 . For people with osteoarthritis, coronary artery disease or congestive heart failure, or cerebrovascular disease, we assigned EOSS stage 3, as in the method of Padwal and colleagues. ${ }^{5}$ EOSS stage 4 denotes end-stage disease; given the small number of patients with this level of disease, we combined EOSS stage 4 with EOSS stage 3.

\section{Dashboard}

In collaboration with the NAPCReN and the Physician Learning Program, we created a prototype for a primary care obesity dashboard using EOSS stages in their data presentation tool. All individuals 18 years of age or older with $\mathrm{BMI}$ of at least 25 are included in the dashboard.

The data presentation tool, created by CPCSSN for both research and quality improvement applications, provides a data visualization overview of all of a physician's patients, including key demographic and clinical characteristics. Physicians can use the data presentation tool to identify patients needing further investigation and intervention. ${ }^{15}$ The EOSS dashboard permits the physician to see all of their patients, collectively and individually, by age, sex, EOSS stage, BMI, time since last visit, distance they live from clinic, medical comorbidities, relevant medications, blood pressure, smoking status and key laboratory values (total cholesterol, low-density lipoprotein, high-density lipoprotein, fasting blood glucose, hemoglobin A1c, estimated glomerular filtration rate, aspartate aminotransferase and alanine aminotransferase).

Because the NAPCReN is a partner project with the Physician Learning Program, family physicians participating in the NAPCReN were provided with a link to their EOSS dashboard, along with information on how they could use the dashboard to improve care for their patients living with obesity.

\section{Statistical analysis}

We used both continuous and categorical BMI values for the analyses. The BMI classes were assigned as class I obesity $(30-34.9)$, class II obesity (35-39.9) and class III obesity $(\geq 40) .{ }^{19}$ We performed bounds-checking of height and weight values to ensure there were no errors in measurement units before calculation of BMI by NAPCReN. We dichotomized sex as male and female.

We used descriptive statistics to describe the population, individual comorbidities and overall EOSS stages by BMI classes. We built ordinary least squares regression models using a stepwise approach to describe the variation in overall EOSS stages, explained by age only, by age and sex, and by age, sex and BMI. Because severity of osteoarthritis could not be determined from the available data, we performed additional analyses to understand how 
it contributed to EOSS stage 3. Although biased point estimates may result when ordinary least squares regression is used to estimate an ordinal integer variable such as EOSS, ${ }^{20}$ we were interested only in describing the amount of explanatory power that age, sex and BMI had in relation to the EOSS stage in this population. Consequently, we have not presented coefficients, because they are unnecessary for the purposes of these analyses. When making a methodologic choice, measures of goodness of fit provide a reasonable estimate ${ }^{21,22}$ with the benefit of considerably greater ease of interpretability. For our descriptive purposes, this, rather than measures of goodness of fit in logistic and ordered logistic regressions, was a reasonable approach..$^{23,24}$

We used SQL Developer (Oracle) to clean the source data. We conducted all analyses with Python 3.4 (Python Software Foundation) and Stata 16 (StataCorp) software.

\section{Ethics approval}

This study received ethics approval from the Health Research Ethics Board (Pr000074666) at the University of Alberta, Edmonton.

\section{Results}

Table 1 presents the descriptive characteristics of the population. In our sample of 23460 patients included in the analysis, the mean age was 54.3 (standard deviation [SD] 17.0) years, 10590 (45.1\%) were male, and the mean BMI was 35.5 (SD 5.5). More than half of the patients $(52.9 \%)$ had overall EOSS stage 2. Figure 2 outlines patients included and excluded in the analysis portion.

\begin{tabular}{|lc|}
\hline \multicolumn{2}{|l|}{ Table 1: Characteristics of patients } \\
\hline & $\begin{array}{c}\text { No. }(\%) \text { of patients } \\
n=23460\end{array}$ \\
\hline Characteristic & $54.3 \pm 17.0$ \\
\hline Age, yr, mean \pm SD & $10590(45.1)$ \\
\hline Sex, male & $35.5 \pm 5.5$ \\
\hline BMI, mean \pm SD & $12767(54.4)$ \\
\hline Obesity classification based on BMI & $6246(26.6)$ \\
\hline Obesity class I (30-34.9) & $4447(19.0)$ \\
\hline Obesity class II (35-39.9) & $610(2.6)$ \\
\hline Obesity class III ( $\geq 40)$ & $4525(19.3)$ \\
\hline Obesity classification based on EOSS & $12405(52.9)$ \\
\hline EOSS stage 0 & $5392(23.0)$ \\
\hline EOSS stage 1 & $528(2.3)$ \\
\hline EOSS stage 2 & \\
\hline EOSS stage 3 & \\
\hline EOSS stage could not be calculated & \\
\hline $\begin{array}{l}\text { Note: BMI = body mass index, EOSS = Edmonton Obesity Staging System, } \\
\text { SD = standard deviation. } \\
{ }^{*} \text { Except where indicated otherwise. }\end{array}$ & \\
\hline
\end{tabular}

\section{Population EOSS stages}

Figure 3 shows the proportion of patients with comorbidity EOSS stages assigned and those with data unavailable. Among those with EOSS stages assigned, the proportion of patients in each EOSS comorbidity stage is illustrated. The range of missing comorbidity data spanned from $11.1 \%$ for hypertension to $17.8 \%$ for dyslipidemia. The proportion of people with obesity who had serious levels of comorbidities with EOSS stage 3 was $14.7 \%$ for osteoarthritis, $8.5 \%$ for coronary heart disease and congestive heart failure, and $2.3 \%$ for cerebrovascular disease.

Table 2 provides the EOSS stages for the 8036 adult patients (13.7\%) for whom BMI data were not available. Of these patients, $4074(50.7 \%)$ had EOSS stage $2(n=2822)$ or EOSS stage $3(n=1252)$.

\section{Dashboard}

The prototype for the primary care dashboard using EOSS stages that we developed is provided in Appendix 2 (available at www.cmajopen.ca/content/9/4/E1141/suppl/DC1).

\section{Variation in EOSS stages: statistical modelling}

In our stepwise ordinary least squares model, age alone described $31 \%$ of the variation in EOSS stages: as age increased, overall EOSS stage also increased. Sex and BMI explained very little of the variation in EOSS stages, together accounting for just over $1 \%$ of the variation (Table 3 ). BMI alone did not drive EOSS stages to any great extent, as illustrated in Figure 4.

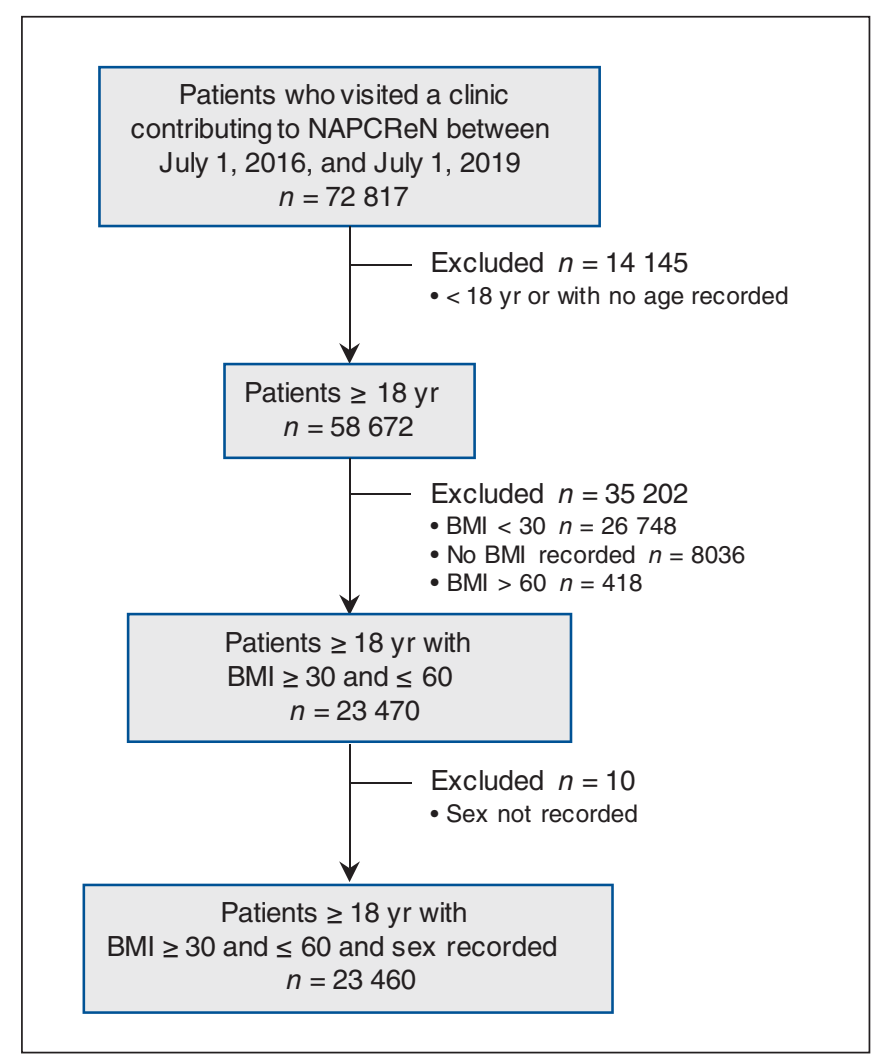

Figure 2: Flow diagram of the inclusion criteria for the patient population. $\mathrm{BMI}=$ body mass index, NAPCReN = Northern Alberta Primary Care Research Network. 


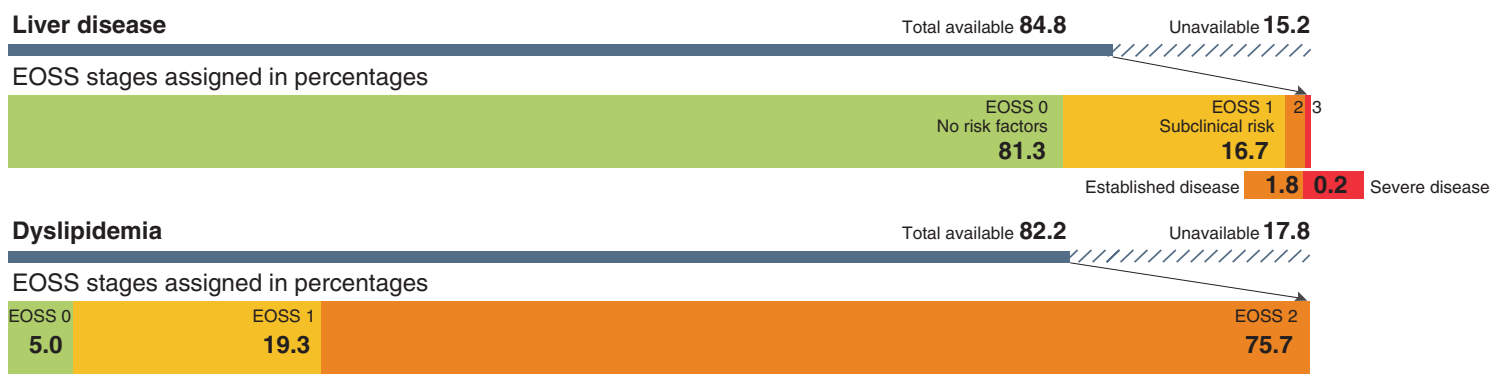

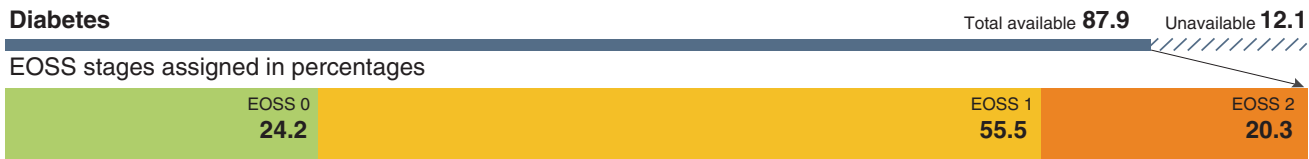

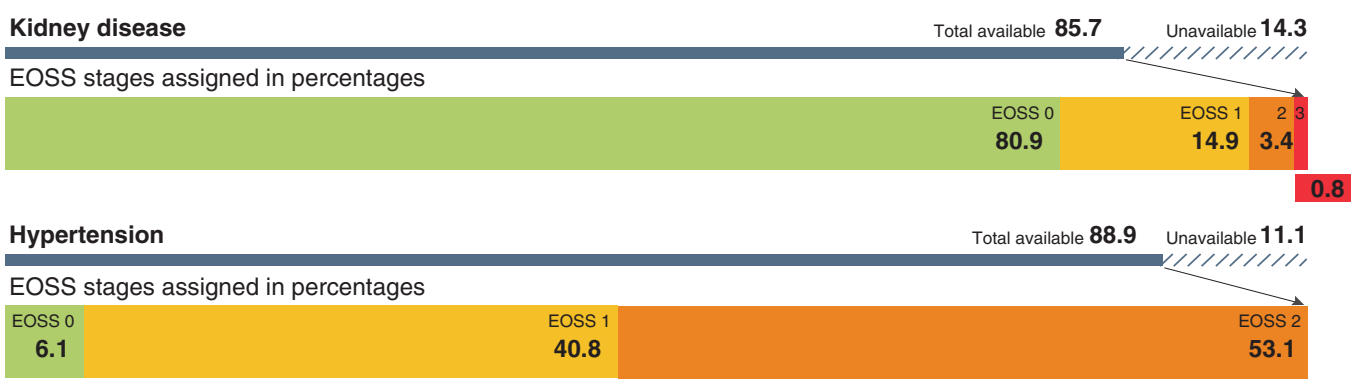

Figure 3: Proportions of patients with data available to assign Edmonton Obesity Staging System (EOSS) comorbidity stage. Among those with data available, the proportion of patients in each EOSS stage is shown.

We could not differentiate disease severity for osteoarthritis, so we conducted further analysis to understand what degree of osteoarthritis drove the EOSS stage. We found that $15.7 \%$ of patients with osteoarthritis had an EOSS stage of 3 for another comorbidity, which suggested that their overall EOSS stage was not being driven by osteoarthritis alone.

\section{Interpretation}

We have shown the feasibility of using primary care data to calculate EOSS stages and have developed a clinical dashboard; these tools are urgently needed to allow clinicians to monitor disease severity and facilitate management in accordance with the 2020 Canadian clinical practice guideline for obesity in adults. ${ }^{2}$ Those working in the field of obesity medicine now recognize the importance of focusing on functional health and organ impairment, rather than body weight exclusively. ${ }^{1}$ Consistent with the literature, we have shown that the EOSS comorbidity-driven approach to obesity provides additional clinical information, which may enhance the usefulness of BMI. Future work will be needed to determine how to incorporate EOSS more seamlessly into routine care, in particular with integration of functional impairment in clinical assessment. This, together with integration of knowledge of the person's life context, will be crucial to working with the patient to create a suitable management plan.,25

Using the dashboard, clinicians can more easily identify patients who may require closer assessment and follow-up, such as those with higher EOSS, those whose EOSS changes, those who are "behind" in chronic disease management and those who have not been seen for follow-up for long periods. The dashboard can also be used as a tool to promote datadriven practice quality improvement initiatives, including support of evidence-based screening for obesity-related comorbidities at the level of the overall practice. We have recently sent individual reports and links to the dashboard to all physicians in the NAPCReN, and our future work will focus on quality improvement in obesity practice.

Given the use of real-world data from primary care electronic medical records, our feasibility study recognizes that variation in clinical practice is warranted and that not all laboratory measurements are indicated for all patients. For example, alanine aminotransferase, one of the measures of liver function, is recommended to screen for nonalcoholic fatty liver disease. ${ }^{2,26}$ However, this recommendation is grade 3, level D, a weak recommendation based on current evidence; as such, not all patients will have been clinically deemed as needing this measure. Conversely, some information was 


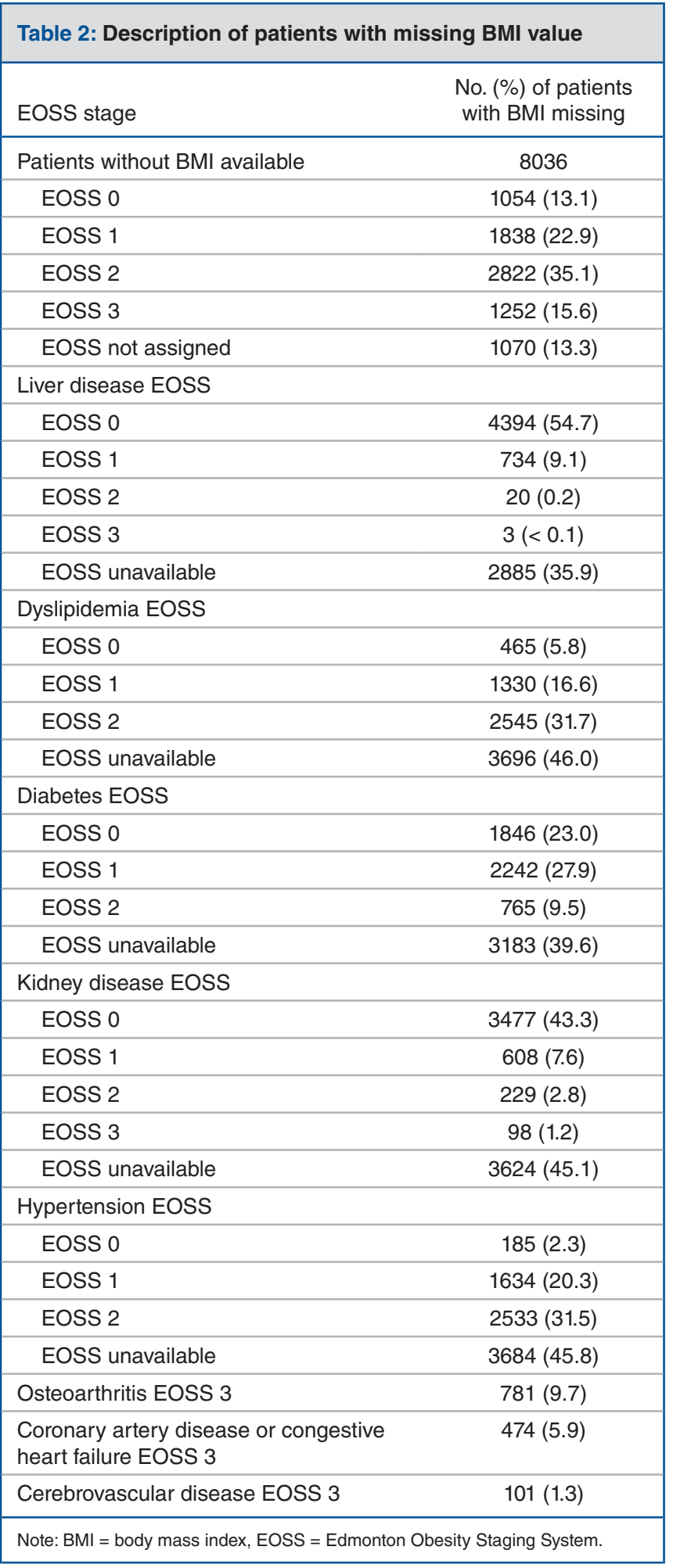

missing that would be clinically relevant. For example, $13.7 \%$ of adult patients did not have height and weight data available to calculate BMI, yet $50.7 \%$ of these had EOSS stage 2 or 3 , values associated with worse clinical outcomes. Despite these realities of the data, we were able to calculate EOSS stages for most patients. The fact that $52.9 \%$ of our adult population

Table 3: Models to determine how much variation in EOSS stages can be attributed to age, sex and BMI*

\begin{tabular}{|lcc|}
\hline Stepwise model† & $\begin{array}{c}R^{2} \text { or \% of variation } \\
\text { in EOSS explained }\end{array}$ & $\begin{array}{c}\text { Increment } \\
\text { in } R^{2}\end{array}$ \\
\hline Model 1: Age only & 31.04 & - \\
\hline Model 2: Age and sex & 31.05 & 0.01 \\
\hline Model 3: Age, sex and BMl & 32.26 & 1.21 \\
\hline
\end{tabular}

Note: $\mathrm{BMI}=$ body mass index, EOSS = Edmonton Obesity Staging System. *Of the total population, 528 were missing or had no EOSS stage and thus were not included in the model; as such, the number of patients used for modelling was 22 932. The stepwise models add variables but do not include interaction effects of the variables.

†ln these models, EOSS is the outcome variable (stage 0 , stage 1 , stage 2 , stage 3 ).

with BMI greater than 30 were in the EOSS stage 2 category identifies a large subset of patients who would benefit from targeted clinical interventions to prevent the development of obesity-related end-organ damage, such as cardiovascular disease or liver disease.

Since publication of the seminal work of Padwal and colleagues in $2011,{ }^{5}$ several studies have examined the usefulness of the EOSS; these have been compiled in a review by Atlantis and colleagues. ${ }^{3}$ Several researchers ${ }^{6,7}$ have confirmed the observation of Padwal and colleagues ${ }^{5}$ that risk of death increases with EOSS stage 3. In patients who have undergone bariatric surgery, EOSS stage 3 was associated with increases in postoperative complications, 30-day mortality and years of life lost. ${ }^{27-29}$ The EOSS stage may also help to predict the chance of cesarean delivery in high-risk nulliparous women with overweight or obesity who undergo induction of labour at term, with a substantial increase in risk observed with EOSS stage $3 .^{30}$

Although the EOSS is the most researched clinical staging system for obesity, several others have been described, including the King's Obesity Staging Criteria, ${ }^{31-33}$ as well as 2 surgery-focused scores, the Obesity Surgery Score ${ }^{34}$ and the Obesity Surgery Mortality Risk Score. ${ }^{35}$ Atlantis and colleagues ${ }^{3}$ also described the various ways in which the EOSS has been operationally defined in studies to date. It is important to note that the broader EOSS typically assesses multiple dimensions of health, including physical symptoms, psychopathology, functional limitations and impairment of well-being. ${ }^{7}$ As has been the case in other studies, ${ }^{5}$ some of these dimensions of health could not be evaluated in our study using existing databases. However, the observation of increased comorbidity burden associated with important patient outcomes is the key clinical point in the care of people living with obesity.

\section{Limitations}

We recognize a number of limitations of our study. The EOSS is an evolving clinical tool with operational definitions that vary across different studies. We adhered to the approach of Padwal and colleagues 5 and recognize that this approach does not encompass physical function or mental health parameters. Future work should focus on assessing the operational definitions of EOSS with these parameters included. 


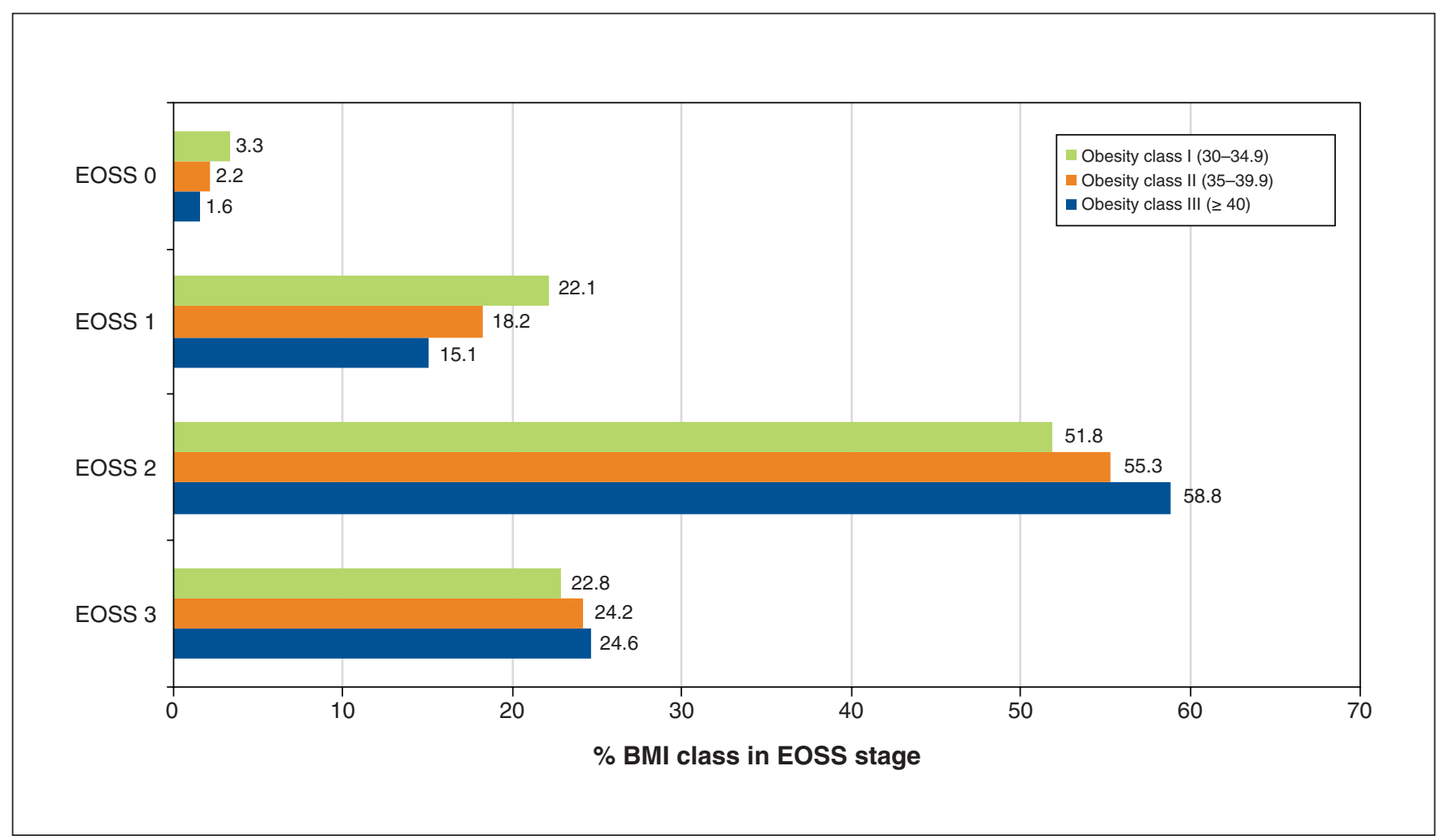

Figure 4: Percentage of patients in each body mass index (BMI) class by overall stage according to the Edmonton Obesity Staging System (EOSS).

In our study, wherever possible, we used validated CPCSSN case definitions to identify and classify patients' comorbidities. However, the CPCSSN has not yet validated some of the comorbidities that were needed for this feasibility study. Future work to expand the list of validated data definitions will enhance this tool. Our collaborative creation of data definitions by clinicians and data scientists, focusing on clinical practice guidelines, appears to be a reasonable first step.

In addition, other than sex and age, data for relevant sociodemographic factors reflecting health determinants, such as income and education level, were not reliably available. Increasing the availability of these variables would help refine factors that may contribute to increasing EOSS.

\section{Conclusion}

Obesity is a prevalent chronic disease in which worse patient outcomes occur at higher clinical stages, as defined by increasing severity of comorbidities. Using the EOSS may enhance clinically important obesity information over using BMI alone. We found that it was feasible to use primary care data to calculate clinical EOSS stages and to develop a clinical obesity chronic disease dashboard. Although data on further dimensions of health and health determinants could be improved in future databases, the information currently available affords an opportunity to intervene to prevent progression of the disease and may help to limit both the health and the economic burdens of obesity.

\section{References}

1. Sharma AM, Campbell-Scherer DL. Redefining obesity: beyond the numbers. Obesity (Silver Spring) 2017;25:660-1.

2. Wharton S, Lau DCW, Vallis $M$, et al. Obesity in adults: a clinical practice guideline. CMA7 2020;192:E875-91.

3. Atlantis E, Sahebolamri M, Cheema BS, et al. Usefulness of the Edmonton Obesity Staging System for stratifying the presence and severity of weightrelated health problems in clinical and community settings: a rapid review of observation studies. Obes Rev 2020;21:e13120.

4. Rueda-Clausen CF, Poddar M, Lear SA, et al. Canadian adult obesity clinical practice guidelines (CPGs): assessment of people living with obesity. Edmonton: Obesity Canada; 2020. Available: https://obesitycanada.ca/guidelines/assessment/ (accessed 2021 July 14).

5. Padwal RS, Pajewski NM, Allison DB, et al. Using the Edmonton obesity staging system to predict mortality in a population-representative cohort of people with overweight and obesity. CMAJ 2011;183:E1059-66.

6. Ejima K, Xavier NA, Mehta T. Comparing the ability of two comprehensive clinical staging systems to predict mortality: EOSS and CMDS. Obesity (Silver Spring) 2020;28:353-61.

7. Kuk JL, Ardern CI, Church TS, et al. Edmonton obesity staging system: association with weight history and mortality risk. Appl Physiol Nutr Metab 2011;36:570-6.

8. Bancej C, Jayabalasingham B, Wall RW, et al. Evidence brief: Trends and projections of obesity among Canadians. Health Promot Chronic Dis Prev Can 2015; 35:109-12.

9. Janssen I. The public health burden of obesity in Canada. Can 7 Diabetes 2013;37:90-6.

10. Twells LK, Gregory DM, Reddigan J, et al. Current and predicted prevalence of obesity in Canada: a trend analysis. CMAF Open 2014;2:E18-26.

11. Tran BX, Nair AV, Kuhle S, et al. Cost analyses of obesity in Canada: scope, quality, and implications. Cost Eff Resour Alloc 2013;11:3.

12. Overweight and obese adults, 2018 [health fact sheet]. Cat no 82-625-X. Ottawa: Statistics Canada; 2019. Available: https://www150.statcan.gc.ca/n1/ en/pub/82-625-x/2019001/article/00005-eng.pdf?st=EmJRdRTc (accessed 2020 June 26).

13. Physician Learning Program [home page]. Edmonton and Calgary: Physician Learning Program. Available: https://www.albertaplp.ca/ (accessed 2021 June 16).

14. Northern Alberta Primary Care Research Network (NAPCReN) [home page]. Edmonton: University of Alberta, Department of Family Medicine, NAPCReN. Available: napcren.ca/ (accessed 2021 Mar. 10). 
15. Canadian Primary Care Sentinel Surveillance Network (CPCSSN) [home page]. CPCSSN; 2020. Available: https://cpcssn.ca/ (accessed 2020 Aug. 5).

16. CPCSSN case definitions: version 2. Canadian Primary Care Sentinel Surveillance Network (CPCSSN); 2019. Available: cpcssn.ca/wp-content/uploads /2019/07/CPCSSN-Case-Definitions-v2.pdf (accessed 2021 June 16).

17. Diabetes Canada Clinical Practice Guidelines Expert Committee. Diabetes Canada 2018 clinical practice guidelines for the prevention and management of diabetes in Canada. Can 7 Diabetes 2018;42(Suppl 1):S1-325.

18. Levin A, Stevens PE. Summary of KDIGO 2012 CKD guidelines: behind the scenes, need for guidance, and a framework moving forward. Kidney Int 2014; 85:49-61.

19. Body mass index - BMI. Copenhagen: World Health Organization, Regional Office for Europe. Available: https://www.euro.who.int/en/health-topics/ disease-prevention/nutrition/a-healthy-lifestyle/body-mass-index-bmi (accessed 2020 Feb. 8).

20. Min H. Ordered logit regression modeling of the self-rated health in Hawai'i, with comparisons to the OLS model. 7 Mod Appl Stat Methods 2013;12:371-80.

21. Peel MJ, Goode MHM, Moutinho LA. Estimating consumer satisfaction: OLS versus ordered probability models. Int 7 Commer Manag 1998;8:75-93.

22. Pohlman JT, Leitner DW. A comparison of ordinary least squares and logistic regression. Obio 7 Sci 2003;103:118-25.

23. Fagerland MW, Hosmer DW. Tests for goodness of fit in ordinal logistic regression models. 7 Stat Comput Simul 2016;86:3398-418.

24. Kapsalis C. Bridging logistic and OLS regression. MPRA Paper 27706. Munich (Germany): University Library of Munich; 2010.

25. Luig T, Elwyn G, Anderson R, et al. Facing obesity: adapting the collaborative deliberation model to deal with a complex long-term problem. Patient Educ Couns 2019;102:291-300.

26. Ginsberg HN, Zhang YL, Hernandez-Ono A. Regulation of plasma triglycerides in insulin resistance and diabetes. Arch Med Res 2005;36:232-40.

27. Chiappetta S, Stier C, Weiner RA; members of StuDoQIMBE of Deutsche Gesellschaft für Allgemein- und Viszeralchirurgie/StuDoQ. The Edmonton Obesity Staging System predicts perioperative complications and procedure choice in obesity and metabolic surgery - a German nationwide registerbased cohort study (StuDoQIMBE). Obes Surg 2019;29:3791-9.

28. Nickel F, de la Garza JR, Werthmann FS, et al. Predictors of risk and success of obesity surgery. Obes Facts 2019;12:427-39.

29. Skulsky SL, Dang JT, Battiston A, et al. Higher Edmonton Obesity Staging System scores are associated with complications following laparoscopic Roux-en-Y gastric bypass. Surg Endosc 2020;34:3102-9.

30. Demsky AN, Stafford SM, Birch D, et al. The Edmonton Obesity Staging System predicts mode of delivery after labour induction. 7 Obstet Gynaecol Can 2020;42:284-92.

31. Valderhaug TG, Aasheim ET, Sandbu R, et al. The association between severity of King's Obesity Staging Criteria scores and treatment choice in patients with morbid obesity: a retrospective cohort study. BMC Obes 2016;3:51.

32. Aasheim ET, Aylwin SJB, Radhakrishnan ST, et al. Assessment of obesity beyond body mass index to determine benefit of treatment. Clin Obes 2011;1:77-84.

33. Aylwin S, Al-Zaman Y. Emerging concepts in the medical and surgical treatment of obesity. Front Horm Res 2008;36:229-59.

34. Casimiro Pérez JA, Fernández Quesada C, Del Val Groba Marco M, et al. Obesity Surgery Score (OSS) for prioritization in the bariatric surgery waiting list: a need of public health systems and a literature review. Obes Surg 2018;28:1175-84.

35. DeMaria EJ, Portenier D, Wolfe L. Obesity surgery mortality risk score: proposal for a clinically useful score to predict mortality risk in patients undergoing gastric bypass. Surg Obes Relat Dis 2007;3:134-40.
Affiliations: Division of Endocrinology and Metabolism, Department of Medicine (Swaleh, Yeung), Edmonton Physician Learning Program (McGuckin, Myroniuk, Manca, Campbell-Scherer, Yeung), Department of Family Medicine (Manca, Campbell-Scherer), Division of Preventive Medicine, Department of Medicine (Lee), and Department of Medicine (Sharma), Faculty of Medicine and Dentistry; School of Public Health (Lee); School of Urban and Regional Planning (Lee); and Alberta Diabetes Institute (Sharma, Campbell-Scherer, Yeung), University of Alberta, Edmonton, Alta.; and Department of Public Health (Myroniuk), University of Missouri-Columbia, Columbia, Mo.

Contributors: Rukia Swaleh and Taylor McGuckin are joint first authors. Donna Manca, Karen Lee, Arya Sharma, Denise CampbellScherer and Roseanne Yeung conceived the project idea. Rukia Swaleh, Taylor McGuckin, Donna Manca, Karen Lee, Arya Sharma, Denise Campbell-Scherer and Roseanne Yeung designed the study methods. Taylor McGuckin and Tyler Myroniuk completed the data analysis. Rukia Swaleh, Taylor McGuckin, Tyler Myroniuk, Denise CampbellScherer and Roseanne Yeung wrote the manuscript, and Rukia Swaleh, Donna Manca, Karen Lee, Arya Sharma, Denise Campbell-Scherer and Roseanne Yeung refined the discussion and conclusion. All of the authors had access to the data and analyses, approved the final version for publication and agreed to be accountable for the work.

Funding: This study was supported by a financial contribution from the Government of Alberta through the Physician Learning Program. The views expressed herein do not necessarily represent official policy of the Government of Alberta. Funding was also provided through a Novo Nordisk Alberta Diabetes (NOVAD) grant, sponsored by the University Hospital Foundation, Novo Nordisk Canada and Alberta Economic Development and Trade.

Content licence: This is an Open Access article distributed in accordance with the terms of the Creative Commons Attribution (CC BY-NC-ND 4.0) licence, which permits use, distribution and reproduction in any medium, provided that the original publication is properly cited, the use is noncommercial (i.e., research or educational use), and no modifications or adaptations are made. See: https://creativecommons.org/licenses/by-nc-nd/4.0/

Data sharing: To request the programming code used to produce the results presented in this manuscript, please contact the Physician Learning Program at the University of Alberta (plp@ualberta.ca). Raw data can be requested from the CPCSSN (cpcssn.ca/join-cpcssn/for-researchers/).

Acknowledgements: The authors would like to acknowledge Brian Forst for his assistance with data extraction and Brock Setchell for his role in both data extraction and manipulation; Matt Taylor, supported by EnACT, for creating the dashboard; and Guillermina Noël for design of Figure 3 and Jordan Tate for design of Figure 1.

Supplemental information: For reviewer comments and the original submission of this manuscript, please see www.cmajopen.ca/content/9/4/ E1141/suppl/DC1. 